\title{
Usages de jeux sérieux pour motiver les étudiants : initiatives d'enseignants et impulsions institutionnelles
}

\author{
MATHIEU MuRATET \\ Sorbonne Université, CNRS, LIP6, F-75005 Paris, France \\ INS HEA, 92150 Suresnes, France, mathieu.muratet@lip6.fr \\ THIBAULT CARRON \\ Sorbonne Université, CNRS, LIP6, F-75005 Paris, France \\ Université Savoie Mont Blanc, 73000 Chambéry, France, thibault.carron@lip6.fr \\ IMAN N'HARI \\ Sorbonne Université, CAPSULE, F-75005 Paris, France \\ iman.nhari@sorbonne-universite.fr \\ BERNOLD HASENKNOPF \\ Sorbonne Université, CNRS, IPCM, F- 75005 Paris, France \\ bernold.hasenknopf@sorbonne-universite.fr \\ BENOIT GROSJEAN \\ P.A.S.T.E.U.R., Département de Chimie, École Normale Supérieure, PSL University, \\ Sorbonne Université, CNRS, F-75005 Paris, France, bgrosjean@chimie.ens.fr \\ LUCAS SIXDENIER \\ P.A.S.T.E.U.R., Département de Chimie, École Normale Supérieure, PSL University, \\ Sorbonne Université, CNRS, F-75005 Paris, France, lucas.sixdenier@ens.psl.eu \\ RODOLPHE VUILLEUMIER \\ P.A.S.T.E.U.R., Département de Chimie, École Normale Supérieure, PSL University, \\ Sorbonne Université, CNRS, F-75005 Paris, France, rodolphe.vuilleumier@ens.psl.eu
}

\section{Résumé}

Les attentes et méthodes de travail des étudiants évoluent au cours du temps. Les enseignants au contact de ces nouvelles générations peuvent souhaiter faire évoluer leurs pratiques, leurs méthodes pédagogiques et expérimenter de nouveaux outils. Nous montrons dans cet article que ces transformations des enseignements sont facilitées avec le soutien et l'appui d'initiatives institutionnelles. Nous présentons deux dispositifs mis en œuvre au sein de Sorbonne Université et structurés autour du thème des jeux sérieux afin d'augmenter la motivation de leurs utilisateurs. Le premier dispositif propose aux participants de jouer à un jeu sérieux alors que le second invite les participants à concevoir leur propre jeu sérieux. De ces expériences, nous illustrons le processus d'innovation pédagogique en œuvre au sein de Sorbonne Université. 


\begin{abstract}
Students' working methods and students' expectations change over time. Dealing with these new student generations, teachers may wish to propose new practices, to try different pedagogical methods and to experiment new tools. We show in this paper that these teaching transformations are facilitated with the support of institutional initiatives. We focus on two experimentations, performed at Sorbonne University, based on serious games to motivate their users. The first experimentation enables users to play a serious game while the second invites participants to design their own serious game. From these experimentations we show how pedagogical innovation is structured in our university.
\end{abstract}

\title{
Mots-clés
}

Motivation, supports institutionnels, jeux sérieux

Keywords

Motivation, institutional support, serious games

\section{Introduction et problématique : une nouvelle génération d'apprenants}

Les générations d'apprenants se succèdent, façonnées par la société et les avancées technologiques. Ainsi, ces nouvelles générations d'apprenants travaillent différemment et ont développé des capacités particulières. Après la génération Y, nommée ainsi pour qualifier d'une part une génération cherchant sans cesse des explications au monde qui l'entoure ( $\mathrm{Y}$ » et « Why » sont des homonymes en anglais) et d'autre part une génération, écouteurs aux oreilles (le «Y » représentant la forme du fil), s’isolant ainsi du monde des études et du travail (Reilly, 2012), les sociologues ont maintenant identifié la prochaine génération : la génération $Z$ ou la génération alpha ou encore nommée également peut-être de manière la plus explicite, la génération $\mathrm{C}$ pour la communication, la collaboration, la connexion et la créativité (McCrindle et Fell, 2020). Si nous nous appuyons sur la littérature en sociologie (Palley, 2012), nos étudiants appartiennent désormais à cette dernière génération : $\mathrm{Z}$. 
L'arrivée et l'identification de nouvelles générations suscitent des préoccupations et des réflexions, que ce soit dans une entreprise, pour leur intégration (Sheahan, 2005) et leur management (Cui et al., 2003), mais aussi dans les sciences de l'éducation où nous cherchons des stratégies pour améliorer l'apprentissage des générations futures (Miller, 2012). Les particularités de ces générations ont été identifiées et analysées à mesure qu'elles progressaient dans les niveaux d'étude. En 2010, Elmore décrit précisément la fin de la génération Y et sent apparaître les spécificités de la suivante (Elmore, 2010) : les étudiants de ces deux générations ont maintenant grandi avec l'ordinateur, Internet et sont immergés dans les nouvelles technologies. Elles sont toujours à la recherche d'innovations et d'épanouissement personnel. En 2016, Seemiller et Grace s'intéressent spécifiquement à leur arrivée dans le supérieur et affinent le profil en marquant les distinctions avec la génération précédente (Seemiller \& Grace, 2016). Par exemple, selon Seemiller et Grace, ces étudiants ne sont plus centrés sur eux-mêmes («me-centric ») mais centrés sur eux au sens de leur groupe («we-centric »). Ainsi, depuis 2013, ces étudiants arrivent dans nos campus et suivent nos programmes universitaires. Dès 2014, des études ont donc été menées spécifiquement sur leur intégration dans le supérieur (Seemiller et Grace, 2017). De plus, habitués à obtenir rapidement des informations, des réponses ou des résultats, ces nouveaux étudiants font preuve d'une certaine impatience, ce qui, dans le monde de l'entreprise, peut être un atout pour progresser rapidement. Ils sont acteurs du principe du «turn over », ils changent sans états d'âme d'entreprises (Reilly, 2012). Ils essaient également de se démarquer des autres générations.

Naturellement, ces changements affectent et continueront d'affecter leur comportement en tant qu'apprenants. Par conséquent, leurs spécificités doivent être prises en compte. Mohr et Mohr mettent en lumière et synthétisent les principales difficultés rencontrées et soulevées par les enseignants (Mohr et Mohr, 2017) dont une difficulté à transmettre les connaissances face à la présence simultanée d'innombrables moyens de communication. Ils notent également pour les étudiants un apprentissage superficiel ou différé (dans le temps ou dans l'espace) et un faible intérêt pour les aspects théoriques et/ou certains modes d'enseignement. Mohr et Mohr proposent ainsi un certain nombre de conseils et d'exemples concrets pour exploiter correctement leurs aptitudes et répondre à ces difficultés : comment les intéresser ? Comment les motiver? Comment les engager dans la tâche d'apprentissage ? Et comment vérifier que cet apprentissage est durable, efficace, satisfaisant ? Ce sont des défis importants qui s'imposent au monde de l'éducation et même à celui de l'entreprise avec la formation tout au long de la vie. 
Il convient toutefois de relativiser ces approches issues des sciences humaines car les descriptions sont souvent bien plus complexes et présentent des perspectives différentes ou des cadres théoriques spécifiques (sociologique, démographique, ethnologique) allant même jusqu'à faire apparaître des contradictions entre elles suivant que l'on se place sous un angle managérial ou sociétal (Attias-Donfut, 1991). Notre position dans cet article consiste à simplement considérer que les générations apprenantes évoluent et qu'il convient de prendre en compte cela dans le contexte de l'apprentissage et de sensibiliser les formateurs en utilisant de nouveaux outils. Face à ces constats ou problèmes, deux approches existent : ramener les étudiants à un fonctionnement que nous maîtrisons bien, ou essayer d'exploiter les nouvelles capacités dont sont prétendument dotées ces générations émergentes en s'intéressant notamment à la formation des néo-enseignants.

La première approche se traduit parfois par des méthodes coercitives, qui consistent par exemple à interdire ou à bloquer les moyens de communication (smartphone, ordinateur portable), qui brouillent le message du professeur et perturbent le centre de l'attention.

La seconde approche consiste à faire évoluer nos pratiques pédagogiques : c'est-à-dire repenser les manières d'enseigner et imaginer de nouveaux supports pour faire travailler les apprenants en utilisant et en « mélangeant» les voies de l'information, les technologies permettant de tirer profit de leurs capacités d'adaptation et d'intégration d'instruments innovants qui suscitent leur intérêt.

La motivation en contexte scolaire est étudiée depuis de nombreuses années. Viau la définit ainsi : « ... état dynamique qui a son origine dans les perceptions qu'un élève a de lui-même et de son environnement et qui l'incite à choisir une activité, à s'y engager et à persévérer dans son accomplissement afin d'atteindre un but» (Viau, 1997, p. 7). Dans son modèle, la performance d'un élève à réaliser une activité correspond aux résultats observables de l'apprentissage et constitue une conséquence de la motivation (elle en est un indicateur). Agir sur la motivation permet indirectement de faire évoluer les performances des étudiants. Dans ce contexte, la motivation est caractérisée par des déterminants et des indicateurs. Les déterminants de la motivation concernent la perception de la valeur d'une activité, la perception de sa compétence à l'accomplir, et la perception de la contrôlabilité de cette activité. Les indicateurs de la motivation sont caractérisés par le choix d'entreprendre une activité, la persévérance, l'engagement cognitif et la performance.

Le travail présenté dans cet article tente d'aborder le problème du point de vue de la seconde approche (consistant à faire évoluer nos pratiques) en vue de favoriser la motivation. Le parti 
pris présenté est de considérer que les transformations réussies d'enseignements reposent sur des initiatives d'enseignants qui ne peuvent émerger et être rendues opérationnelles sans des impulsions et des soutiens institutionnels. Nous illustrons ce parti pris dans le contexte de Sorbonne Université qui favorise l'émergence de nombreuses initiatives pédagogiques ; nous allons en présenter deux exemples. Le premier est un jeu sérieux de type escape game traitant du thème de la pédagogie. Ce jeu a été exploité lors de la formation des nouveaux enseignantschercheurs recrutés à Sorbonne Université. Ce jeu avait un double objectif : (1) sensibiliser ces nouveaux enseignants-chercheurs à différentes notions relatives à la pédagogie, et (2) leur donner à voir et à manipuler un jeu sérieux afin de les inciter à penser leurs pratiques professionnelles au travers de ressources pédagogiques susceptibles de motiver les nouvelles générations d'étudiants.

Le second exemple n'est pas un jeu sérieux mais invite les apprenants à concevoir et réaliser leur propre jeu sérieux. Ce dispositif pédagogique s'appuie d'une part sur certains aspects de la classe inversée et d'autre part sur une approche par projets afin d'initier au code informatique des étudiants de première année suivant un parcours Biologie-Géoscience-Chimie.

De manière plus générale, notre objectif est de montrer que nous pouvons nous appuyer sur des innovations constantes portées par des enseignants et soutenues par des services spécifiques de l'université. Dans un premier temps, nous décrirons les deux dispositifs employés dans des domaines d'enseignement différents au sein de Sorbonne Université. Après avoir présenté quelques éléments méthodologiques sur la mise en œuvre de ces deux dispositifs, nous détaillerons dans la quatrième partie les retours d'expérience. La partie suivante proposera une discussion des résultats au regard du modèle de la motivation en contexte scolaire de Viau (Viau, 1997). Nous terminerons cette cinquième partie par une illustration schématique du processus d'innovation pédagogique au sein de Sorbonne Université permettant aux enseignants de proposer des enseignements s'écartant d'une approche transmissive classique. 


\section{Contexte}

\subsection{E-LearningScape un jeu sérieux sur le thème de la pédagogie}

E-LearningScape ${ }^{1}$ est une adaptation du jeu LearningScape conçu par SAPIENS ${ }^{2}$ et le CRI ${ }^{3}$. La version originale consistait en un escape game grandeur nature traitant du thème de la pédagogie ${ }^{4}$. Dans cette première version du jeu, les participants (en équipe de 4) jouent le rôle de marchands de sable immergés dans le rêve de Camille, une jeune enseignante-chercheuse à la veille de son premier enseignement. Leur défi consiste à aider Camille à structurer sa pensée durant son sommeil par la résolution d'énigmes sur le thème de la pédagogie.

Bien que ce format « grandeur nature » (salles aménagées, décorées et équipées de matériels tels que des tableaux blancs, des cadenas, des clés, des bureaux...) soit particulièrement apprécié des participants, la contrainte principale de ce format est le passage à l'échelle. En effet, déplacer l'escape game et en créer des copies pour pouvoir faire jouer plusieurs dizaines de participants simultanément nécessite un investissement important en temps et en équipement. C'est pour répondre à cette contrainte qu'E-LearningScape a été développé par Sorbonne Université à travers l'équipe MOCAH ${ }^{5}$ du LIP6 6 , le projet Play@SU et CAPSULE7 . E-LearningScape reprend donc le thème et l'atmosphère du jeu original mais le transforme en un jeu hybride mêlant réel et virtuel. Les concepteurs de ce jeu ont choisi de maintenir certains éléments du jeu dans le monde réel afin d'encourager la collaboration entre les joueurs. ELearningScape peut donc être joué par 2 à 5 joueurs autour d'un ordinateur. Les joueurs évoluent dans un univers virtuel, découvrent des fragments de rêve leur donnant accès à du

${ }^{1}$ E-LearningScape est un jeu open-source téléchargeable à l'adresse suivante : $\underline{\text { https://github.com/Mocahteam/E- }}$ LearningScape, consulté le 15/02/2021.

2 SAPIENS : Service d'Accompagnement aux Pédagogies Innovantes et à l'Enseignement Numérique de Sorbonne Paris Cité.

${ }^{3}$ CRI : Centre de Recherche Interdisciplinaire.

${ }^{4}$ LearningScape : https://sapiens-uspc.com/creer-un-escape-game-pedagogique-dossier-learningscape/, consulté le $15 / 02 / 2021$.

${ }^{5}$ MOCAH : Modèles et Outils en ingénierie des Connaissances pour l'Apprentissage Humain.

${ }^{6}$ LIP6 : Laboratoire d'Informatique de Paris 6.

${ }^{7}$ CAPSULE : Centre d'Accompagnement pour la Pédagogie et SUpport à l'Expérimentation. 
matériel dans le monde réel. Ils résolvent alors des énigmes à l'intérieur et à l'extérieur du jeu vidéo, ces deux facettes s'alimentant mutuellement. Le déploiement du jeu est donc simplifié, il consiste à installer le jeu sur plusieurs ordinateurs et préparer pour chaque poste de travail un ensemble d'enveloppe contenant les documents utiles à la résolution des énigmes.

Outre l'objectif premier de cette ressource, à savoir sensibiliser les joueurs à des questions relatives à la pédagogie, ce jeu avait comme second objectif de donner à voir aux participants une ressource innovante mêlant réel et virtuel comme une invitation à penser leurs futurs enseignements autrement que par des modalités transmissives et eux-mêmes imaginer des ressources pédagogiques susceptibles de motiver les nouvelles générations d'étudiants.

\subsection{Initiation à la programmation par la conception de jeu sérieux de chimie}

Sorbonne Université propose aux étudiants de première année l'unité d'enseignement ARE (Atelier de Recherche Encadrée). Cette unité d'enseignement a vocation à permettre aux étudiants inscrits dans un portail de formation de travailler des disciplines complémentaires. Les ARE correspondent à une forme d'apprentissage basée sur la recherche (Research-Based Learning) (Healey et Jenkins, 2009) qui mettent en exergue le travail en autonomie et en groupe afin de s'approprier une thématique scientifique, mener un raisonnement dans le domaine, restituer le processus de réflexion et les conclusions qui en découlent. Cette unité d'enseignement compte pour $1 / 5$ du second semestre de première année de licence (6 ECTS) et représente donc une part importante de la formation. Cette unité d'enseignement est obligatoire pour tous les étudiants de première année, soit plus de 1500 étudiants chaque année.

Ce canevas de formation offre donc l'opportunité aux enseignants d'expérimenter de nouvelles méthodes et de différencier leurs enseignements. Ainsi, sont proposés des ateliers combinant des disciplines proches comme Informatique/Mathématique ou Physique/Chimie et d'autres mêlant des disciplines plus éloignées comme Géoscience/Sport, Chimie/Informatique ou Physique/SHS. Dans chaque cas, les enseignants sont pleinement acteurs de leurs approches pédagogiques et sont libres d'expérimenter des approches par projets, des classes inversées, d'adapter leurs évaluations ou de suivre des enseignements plus classiques alternant cours/TD/TP.

Dans le contexte des ARE, des enseignants ont proposé l'ARE ChemCraft. Les liens suivants illustrent quelques exemples de jeux réalisés par les étudiants lors des années précédentes :

- Année universitaire 2014-2015 : https://scratch.mit.edu/studios/2069468/ 
- Année universitaire 2015-2016 : https://scratch.mit.edu/studios/2069467/

- Année universitaire 2016-2017 : https://scratch.mit.edu/studios/4471281/

- Année universitaire 2017-2018: https://scratch.mit.edu/studios/5752753/

- Année universitaire 2019-2020 : https://scratch.mit.edu/studios/27509928/

Ainsi, contrairement à E-LearningScape où les apprenants jouent à un jeu sérieux et apprennent de leur expérience de jeu, ici les étudiants sont positionnés en tant qu'expert de leur domaine de compétence et doivent réaliser leur propre jeu. Cette approche inversée leur demande finalement de se documenter et de maîtriser leur problématique en vue de pouvoir l'exprimer sous la forme d'un jeu.

Afin de permettre aux étudiants d'arriver à cet objectif final, l'ARE ChemCraft est structuré en trois parties. La première (1 séance de 4 h) consiste à permettre aux étudiants d'expérimenter un jeu sérieux traitant du thème de la programmation : le jeu Prog\&Play . L'objectif ici est double : illustrer les problématiques inhérentes aux jeux sérieux (motivation intrinsèque, progression de la difficulté, analyse de traces...) et donner une expérience ludique de la programmation à des étudiants potentiellement peu intéressés par cette discipline car ayant choisi de suivre un parcours où l'informatique disciplinaire est mineure. Comme ces étudiants sont pour la plupart novices en programmation, les enseignants ont choisi d'utiliser le jeu Prog\&Play avec l'environnement de programmation Scratch.

Par la suite, dans la deuxième partie ( 2 séances de $2 \mathrm{~h}$ ), les étudiants travaillent sur des articles scientifiques de chimie pour choisir la problématique qu'ils traiteront dans leur jeu et sont initiés à la méthodologie de conception des 6 facettes (Marne et al., 2011) (1 séance de $2 \mathrm{~h}$ ). La troisième partie de l'ARE est la plus importante (10 séances de $2 \mathrm{~h}$ ) et permet aux étudiants avec une approche par projet de réaliser un travail collaboratif afin de produire le jeu sérieux en Scratch et de le défendre devant les autres participants à l'ARE lors de la dernière séance de restitution des travaux réalisés.

\section{Méthode}

E-LearningScape a été déployé à cinq reprises. La première session de jeu a été organisée lors de la formation des nouveaux enseignants-chercheurs recrutés à Sorbonne Université et les sessions suivantes avec les doctorants de Sorbonne Université ayant des charges d'enseignement. L'organisation des séances a suivi le format suivant : 1 heure de jeu par groupe de 4 joueurs suivi d'une session de débriefing de 30 min sur l'expérience de jeu et les concepts 
abordés. Toutes ces séances de jeu ainsi que les séances de débriefing ont été animées par un même ingénieur pédagogique.

Le retour d'expérience présenté dans cet article concerne la première session de jeu qui a été organisée avec les nouveaux enseignants-chercheurs recrutés à Sorbonne Université. Le panel de participants contenait 22 femmes et 14 hommes, âgés de 25 à 40 ans. Ces apprenants appartiennent ou vont naturellement être confrontés aux générations dont on parlait en introduction (nous nous situons dans ce cas plus dans l'approche « formation de formateurs à ces nouveaux outils »). Ces enseignants-chercheurs nouvellement recrutés venaient de cultures scientifiques très différentes (16 provenant de la faculté des sciences et de l'ingénierie et 20 de la faculté des arts et des humanités). La session de jeu était positionnée lors de la première journée de formation après l'accueil et l'ouverture des deux jours de formation. Lors de cette session de jeu, 9 groupes ont été organisés répartis en 4 salles. Outre l'ingénieur pédagogique en charge d'animer la séance, deux observateurs prenaient des notes sur les interactions entre les participants.

Concernant l'ARE ChemCraft, il a été mis en œuvre la première fois lors de l'année universitaire 2014-2015 (il est depuis reproposé chaque année aux étudiants). Cet ARE accueille donc chaque année une trentaine d'étudiants inscrits en première année de licence dans un parcours intitulé «Biologie-Géoscience-Chimie ». Dans ce parcours, l'informatique est donc une discipline mineure. Les étudiants expriment des vœux en début de semestre sur les différents ateliers auxquels ils souhaiteraient participer sur la base d'une description courte de chaque atelier. Cette description présente le contexte de l'atelier, les objectifs visés et les modalités d'évaluation. Les groupes sont donc constitués pour permettre à chaque étudiant d'obtenir l'un de ses choix mais le premier choix n'est pas toujours respecté (ce choix est opéré par les responsables des ARE, les enseignants porteurs des ateliers ne participent pas à cette répartition des étudiants).

Dans l'ARE ChemCraft, les étudiants travaillent en groupe de 3 à 4 personnes pour concevoir et réaliser un jeu sérieux sur la problématique de chimie de leur choix. L'encadrement est réalisé par trois enseignants (un enseignant-chercheur en chimie, un doctorant en chimie et un enseignant-chercheur en informatique). Le retour d'expérience présenté dans la section suivante se base sur les notes des enseignants participant à l'encadrement de cet ARE et sur les retours des étudiants réalisés à travers les rapports et les présentations de leurs jeux en fin de semestre. 


\section{Analyse des deux dispositifs}

\subsection{E-LearningScape}

L'annonce en début de formation consistant à commencer la journée de formation par un jeu vidéo a permis, dans un premier temps, de capter l'attention des participants. En fonction de leurs rapports passés aux jeux vidéo certains participants ont manifesté un a priori plus ou moins positif. Certains exprimaient qu'ils n'étaient pas du tout « jeu vidéo » et d'autres au contraire manifestaient par leur attitude une posture très positive (larges sourires, clins d'œil entre participants...). Ces manifestations n'ont pas été explicitement verbalisées à l'attention de l'ingénieur pédagogique en charge de mener la séance mais ont été notées par les deux observateurs de la séance à partir de leurs observations des communications verbales et non verbales entre les participants. Ces simples observations parcellaires (gardant à l'esprit la taille limitée de l'échantillon concerné) semblent dénoter une acculturation hétérogène aux jeux vidéo, notamment pour ces enseignants-chercheurs appartenant à des générations différentes (nous rappelons que les participants étaient âgés de 25 à 40 ans), les jeux vidéo et donc les jeux sérieux restent des artefacts porteurs de modalités d'interactions pouvant potentiellement mettre des utilisateurs en difficulté. Dans le cadre d'E-LearningScape, nous pensons que la dimension collaborative a contribué à contrôler ce risque lié aux difficultés d'interaction. Les participants, répartis en groupes de 3 à 4 joueurs, se sont organisés de manière autonome. Des rôles ont émergé en fonction des compétences de chaque participant comme le « contrôleur du jeu » qui gérait le déplacement dans l'environnement virtuel ou « le préposé aux enveloppes » qui gérait le stock de fragments de rêve. Dans certains groupes, ces rôles étaient moins bien définis (plusieurs participants gérant la même tâche) ou étaient mouvants au cours du temps (passage de la souris d'un participant à l'autre).

Pour autant, la collaboration n'a pas été efficace dans tous les groupes. Certains participants surinvestis s'impliquaient dans toutes les tâches et ne laissaient que peu de place aux autres participants. Ces situations ont été reprises lors de la phase de débriefing pour permettre aux participants de porter un regard réflexif sur les interactions interpersonnelles qu'ils venaient de vivre et qu'ils rencontreront dans leurs futurs enseignements lorsqu'ils proposeront des activités collaboratives à leurs étudiants.

D'un point de vue ludique, E-LearningScape semble couvrir une large palette de profil de joueur (Nacke et al., 2011). Les seekers apprécient l'environnement onirique et le scénario proposé, les masterminds sont moteurs dans la résolution des énigmes, les conquerors poussent 
à essayer de terminer le jeu en un temps limité, les socialisers participent à la cohésion du groupe et les achievers apprécient de fouiller et rechercher le moindre petit indice dans le jeu. Seuls les profils à dominante survivor et daredevil trouvent peu de mécanique de jeu permettant de satisfaire leurs attentes ludiques. Néanmoins un profil de joueur n'étant pas centré sur une et une seule classe, tous les participants se sont impliqués dans la session de jeu et nous n'avons pas relevé d'abandon ou de décrocheur au sein des groupes.

La question de la gestion du temps a été un fort levier motivationnel noté par les participants. L'ensemble des groupes avait à cœur de terminer le jeu et bien qu'aucune compétition n'ait été instaurée entre les groupes lors du lancement de la session du jeu, certains participants cherchaient à comparer le temps qu'ils avaient mis à terminer le jeu avec les temps des autres groupes. Mais cette comparaison était toutefois biaisée car, bien que les joueurs ne s'en soient pas rendu compte, une charge de travail importante pour l'ingénieur pédagogique lors de la session de jeu était de veiller à ce que la progression des différents groupes ne diverge pas trop. L'objectif étant qu'un maximum de groupes finisse dans un intervalle de temps restreint afin d'éviter aux premiers groupes de trop attendre avant la phase de débriefing. L'ingénieur pédagogique intervenait donc régulièrement au sein des groupes et de manière non équitable afin de donner des indices et aiguiller les groupes en retard.

Cette première expérimentation ainsi que les suivantes ont permis de faire évoluer le jeu. Un premier travail a été mené sur l'adaptation des contenus du jeu à un autre contexte et sur la mise en place de feedbacks en temps réel pour aider les groupes retardataires (Muratet et al., 2020). Le dispositif de suivi intégré au jeu vise à différencier les aides automatiques en fonction de la progression des joueurs et décharger pour partie l'animateur de cette tâche. Un second travail a porté sur l'accessibilité du jeu pour les joueurs porteurs de troubles sensoriels (Garbarini et Muratet, 2020).

L'entretien, mené avec l'ingénieur pédagogique ayant animé les séances, souligne l'importance de la phase de débriefing pour permettre aux joueurs et futurs enseignants d'appréhender le fondement des énigmes qu'ils ont résolues et d'ancrer l'apprentissage dans le réel. C'est un moment d'échange et d'approfondissement de certaines notions traitées dans le jeu et de réflexion sur la ressource pédagogique qu'ils venaient de tester et de ce qu'ils pourraient envisager dans leurs propres enseignements. Sans cette phase de débriefing, les joueurs risqueraient de ne pas apprécier le réel contenu du jeu, mais seulement d'en retenir les éléments de surface. On parle aussi d'ancrer dans le réel ce qui a été appris dans le monde virtuel. 
La force et la valeur ajoutée du jeu se trouvent dans sa dimension collaborative dans le monde réel et sa transposition dans un monde narratif virtuel qui plonge le joueur dans un processus réflexif sur sa manière d'aborder l'enseignement.

\subsection{ChemCraft}

L'ARE ChemCraft est structuré autour de deux domaines disciplinaires, l'Informatique et la Chimie. Le groupe est constitué d'étudiants hétérogènes. Certains sont motivés par cette double compétence Info-Chimie, d'autres sont attirés par l'objectif de l'ARE qui consiste, nous le rappelons, à concevoir et réaliser un prototype de jeu sérieux. Enfin, d'autres suivent cet atelier par défaut faute de n'avoir pu participer à leurs premiers choix (voir section 3). Cette hétérogénéité est un paramètre important pour le travail collaboratif demandé aux étudiants et la répartition des rôles au sein des groupes. La réalisation d'un prototype de jeu sérieux demande aux étudiants de développer plusieurs compétences : maîtriser le domaine de la chimie qu'ils souhaitent traiter dans leur jeu, approfondir leurs compétences en programmation, équilibrer la dimension ludique et la dimension sérieuse de leur jeu, découvrir des logiciels de retouche d'images, planifier un projet (définir les tâches et le rôle de chacun), réaliser des documents écrits et des présentations.

Les étudiants sont libres de s'organiser comme ils l'entendent. Contrairement au jeu ELearningScape où les interactions interpersonnelles sont d'ordre collaboratif (chaque participant pouvant facilement interchanger leurs rôles au cours du jeu), les interactions sociales dans le cadre de l'ARE ChemCraft sont plus complexes. La phase de conception est majoritairement collaborative, souvent sous l'impulsion d'un des membres du groupe, les étudiants définissent un sujet et l'affinent collégialement. La phase de réalisation est quant à elle plutôt d'ordre coopératif où chaque étudiant en fonction de ses atouts s'empare d'une partie du projet (scénarisation du jeu, progression de la difficulté, création des assets graphiques, programmation des mécaniques de jeu, documentation...).

Plusieurs groupes ont rencontré des difficultés pour organiser leur projet, les situations les plus critiques se sont posées lorsque les leaders sur la facette «Chimie » considéraient ceux qui s'étaient positionnés sur la partie informatique comme de simples prestataires de services qui devaient réaliser leurs demandes. La communication entre les membres du groupe était donc déséquilibrée, il n'y avait plus d'échange. Certains étudiants ont souhaité en cours de semestre changer de groupe, les enseignants ont alors réinstauré un dialogue entre les membres du groupe notamment pour les inviter à mieux interpréter et considérer les remarques des uns et des autres, 
par exemple pour comprendre les difficultés techniques rencontrées pour implémenter une mécanique de jeu et en imaginer d'autres plus faciles à intégrer.

Cet ARE est reconduit chaque année depuis 2014 et rencontre un réel succès auprès des étudiants. Ils soulignent l'intérêt d'être positionnés en tant qu'experts de leur domaine de compétence et la stimulation liée au travail en groupe. Les étudiants sont fiers de présenter leur travail en fin de semestre et ont le sentiment d'avoir progressé à la fois dans leur discipline principale, la chimie, en ayant travaillé en profondeur la thématique traitée dans leur jeu, mais aussi en informatique. Certains étudiants ont même exprimé le sentiment d'être réconcilié avec cette discipline mineure pour eux : « je ne pensais pas être capable de réaliser un jeu tel que celui que nous avons fait», «j'ai eu des cours de programmation au premier semestre et je n'avais jamais compris à quoi pouvait bien servir une boucle, là j'ai compris ».

Pour les enseignants, ils notent un réel investissement des étudiants : certains viennent volontairement aux séances qui ne leur sont pas réservées et doublent ainsi leur temps de présence aux travaux pratiques ; d'autres réalisent des ressources non demandées (trailer vidéo de présentation de leur jeu) ; enfin, certains groupes ont poursuivi le développement de leur jeu au-delà de la période d'évaluation.

Chaque itération de l'ARE amène les enseignants à réviser l'organisation des enseignements. Des modifications ont été apportées au fil des années pour demander aux étudiants des rendus intermédiaires ou pour associer la bibliothèque universitaire afin d'enrichir les contenus avec de la recherche documentaire.

\section{Discussion}

Comme annoncé en introduction, nous nous basons sur le modèle de la motivation en contexte scolaire de Viau (Viau, 1997), pour discuter des déterminants et des indicateurs de la motivation des apprenants dans les deux situations présentées et s'en servir de grille d'analyse de ces deux initiatives.

\subsection{Déterminants de la motivation}

\subsubsection{Perception de la valeur d'une activité}

Un premier déterminant dans le modèle de Viau est la perception de la valeur d'une activité. C'est un jugement que l'apprenant porte sur l'utilité d'une activité en vue d'atteindre des buts qu'il poursuit. Ces buts peuvent être classés en deux grandes catégories : (1) les buts sociaux concernent la relation qu'un apprenant établit avec ses pairs et avec l'enseignant ; (2) les buts 
scolaires incluent les buts d'apprentissage (ceux que nous poursuivons lorsque nous accomplissons une activité pour acquérir des connaissances - motivation intrinsèque) et les buts de performance (ceux que nous poursuivons lorsque nous voulons réussir une activité pour que les autres nous estiment et nous reconnaissent ou encore pour obtenir une récompense, des félicitations, etc. - motivation extrinsèque).

Concernant E-LearningScape, les participants ont été, dans un premier temps, motivés par le but social et la perspective de réaliser une activité ludique en petit groupe. Le but d'apprentissage était quant à lui beaucoup plus modeste, les participants n'ayant eu que très peu d'information sur l'objectif du jeu avant de commencer la session. Concernant le but de performance, aucun objectif et aucune évaluation n'étaient attendus par l'ingénieur pédagogique en charge de la session de formation.

Concernant ChemCraft, la dimension sociale était également présente, mais les buts d'apprentissage et de performance étaient quant à eux bien mieux perçus par les étudiants. En effet, chaque groupe devait produire un certain nombre de ressources (prototype fonctionnel du jeu, rapport écrit, soutenance orale) pris en compte dans leur évaluation. Le levier de motivation extrinsèque était donc prégnant dans ce contexte.

\subsubsection{Perception de sa compétence à accomplir une activité}

Un deuxième déterminant porte sur la perception de sa compétence à accomplir une activité, c'est une perception de soi par laquelle la personne, avant d'entreprendre une activité qui comporte un degré élevé d'incertitude quant à sa réussite, évalue ses capacités à l'accomplir de manière adéquate. Selon Bandura (Bandura, 1986), cette perception provient de quatre sources :

(1) les performances antérieures qui correspondent aux succès et échecs passés d'un apprenant ;

(2) l'observation d'autres personnes à exécuter une activité. Observer un pair influence davantage la perception qu'un apprenant a de sa compétence que le fait d'observer un professeur ; (3) la persuasion dont le but est de convaincre un apprenant de ses capacités à accomplir une activité ; (4) les réactions physiologiques et émotives sont également une source de la perception qu'un apprenant a de sa compétence.

Concernant E-LearningScape, les deux observateurs de la séance ont relevé des disparités au sein du groupe. Certains participants ont manifesté des postures très positives (larges sourires, clins d'œil entre participants...) alors que d'autres étaient plus inquiets quant à leurs capacités à interagir avec un jeu vidéo. Cette perception de leur compétence à accomplir l'activité proposée devait être influencée par leurs expériences antérieures avec les jeux vidéo (qui, pour 
certains, devaient être inexistantes). Néanmoins, la perspective de réaliser l'activité en groupe a rassuré les moins à l'aise qui pouvaient s'appuyer sur les compétences de joueurs plus expérimentés. Ces participants, qui se mettaient eux-mêmes dans une posture de suiveur, « Moi, c'est pas du tout mon truc, je te laisse contrôler l'ordinateur... », ont donc pu prendre part à l'activité notamment en participant à la résolution des énigmes mais ne se sentaient pas en mesure de pouvoir projeter ce type d'expérience dans leurs enseignements, n'ayant pas euxmêmes développés les schèmes d'interaction avec ces types d'artefacts.

Concernant ChemCraft, les enseignants n'ont pas observé d'apprenants qui, en début de formation, exprimaient un sentiment d'incapacité à accomplir le cahier des charges annoncé (concevoir leur propre jeu sérieux). La tendance était plutôt inversée avec des groupes d'étudiants qui surestimaient leurs capacités de travail et définissaient des projets beaucoup trop ambitieux par rapport à leurs compétences. Les enseignants jouaient donc un rôle d'accompagnement et de suivi important pour aider chaque groupe à définir le périmètre de leur projet afin qu'il soit suffisamment consistant pour valider l'UE sans prendre des risques démesurés.

\subsubsection{Perception de contrôlabilité de l'activité}

Le dernier déterminant porte sur la perception de la contrôlabilité d'une activité, c'est la perception qu'un apprenant a du degré de contrôle qu'il possède sur le déroulement et les conséquences d'une activité qu'on lui propose de faire.

Concernant E-LearningScape, le jeu met les apprenants en situation d'agir avec un contrôle total de la simulation sans part d'aléatoire. Toutefois, les joueurs bien qu'étant acteurs de la résolution du jeu, ne sont pas dans une démarche créative. En revanche pour ChemCraft, le cœur de l'enseignement porte sur la création d'une ressource qui, par incidence permet aux apprenants d'approfondir des notions disciplinaires en Chimie et en Informatique. Les apprenants ont donc un grand contrôle sur leur projet. Seule la technologie pour prototyper leur jeu est imposée (Scratch). Quelques étudiants ont exprimé le souhait de pouvoir prototyper leur jeu avec un autre langage de programmation comme le Python. Ils avaient le sentiment que Scratch allait les limiter dans leur créativité et que Python leur donnerait plus de contrôle sur leur projet. Après quelques échanges avec leurs enseignants, ils ont finalement accepté d'utiliser l'environnement Scratch plus adapté à la réalisation d'un prototype fonctionnel. Ainsi, l'enseignant ne doit pas totalement s'effacer pour être à même de réguler la multitude d'idées qui pourraient émerger au sein des groupes. 


\subsection{Indicateur de la motivation}

Les indicateurs de la motivation selon le modèle de Viau sont au nombre de quatre : le choix d'entreprendre une activité, la persévérance à réaliser l'activité, l'engagement cognitif et la performance.

Concernant le choix d'entreprendre l'activité, cet indicateur n'a de sens que pour l'ARE ChemCraft. En effet, pour E-LearningScape, les participants n'avaient aucun choix, ils étaient obligés de participer aux journées de formation et de jouer au jeu. En revanche, pour ChemCraft les étudiants devaient formuler des choix parmi l'ensemble des ateliers proposés. Même si les étudiants n'ont pas eu leur premier choix en raison du nombre limité de place par ARE, ils ont participé néanmoins à un atelier qui les intéressait.

Concernant la persévérance, les deux situations ont capté l'attention des participants. Pour $E$ LearningScape, aucun membre de groupe n'a abandonné la partie, même les groupes qui avaient rencontré des difficultés et avaient pris du retard ne voulaient pas arrêter de jouer à l'issue de l'heure de jeu pour rejoindre la séance de débriefing avec les autres participants. L'ingénieur pédagogique a dû insister lourdement pour qu'ils acceptent d'abandonner leur partie. Pour ChemCraft qui était réalisé sur un temps beaucoup plus long (10 séances de $2 \mathrm{~h}$ uniquement pour la partie conception et développement du jeu), la persévérance s'est manifestée sur au moins trois dimensions : (1) certains étudiants venaient volontairement aux séances qui ne leur étaient pas réservées et doublaient ainsi leur temps de présence aux travaux pratiques ; (2) d'autres ont réalisé des ressources non demandées (trailer vidéo de présentation de leur jeu) ; enfin, (3) certains groupes ont poursuivi le développement de leur jeu au-delà de la période d'évaluation. Bien sûr, tous les groupes n'étaient pas autant investis et certains se sont simplement contentés de réaliser le travail demandé.

Concernant l'engagement cognitif, les apprenants ont développé des stratégies d'organisation avec des répartitions de rôles au sein des groupes («contrôleur du jeu » et "préposé aux enveloppes » pour E-LearningScape et « expert Chimie » et «expert Informatique » pour l'ARE ChemCraft). L'intensité de l'engagement a même provoqué des tensions dans certains groupes de l'ARE ChemCraft comme nous l'avons évoqué précédemment. Ces difficultés ont été dépassées en amenant les étudiants à développer de nouvelles stratégies métacognitives afin de prendre conscience de leurs fonctionnements, des stratégies utilisées pour réguler leurs façons de travailler, de revoir l'organisation du temps de travail et la formalisation des décisions prises. 
Concernant la performance, cet indicateur s'applique principalement à l'ARE ChemCraft qui intègre un volet évaluation. Chaque groupe devait à l'issue du semestre rendre un rapport écrit de leurs travaux et défendre leur projet à l'oral devant les autres étudiants de la promotion. Il est toutefois intéressant de noter que même si pour E-LearningScape aucun objectif de performance n'était annoncé aux participants, une forme de compétition inter-groupe s'est tout de même instaurée à l'issue de la session de jeu où les participants comparaient les temps qu'ils avaient mis pour finir le jeu, signe qu'un but de performance implicite avait émergé de la situation proposée.

\subsection{Synthèse}

Même si parfois les interactions entre les participants ont suscité de vifs échanges, elles ont toujours participé à engager les apprenants dans la tâche qui leur était confiée. L'implication des équipes pédagogiques (enseignants, ingénieurs pédagogiques) n'en est toutefois pas réduite et reste fondamentale pour apporter la bonne notion au bon moment ou pour fournir une aide contextualisée pertinente (Mohr et Mohr, 2017). Ce changement de posture de l'enseignant moins dans le transmissif et plus dans l'accompagnement individualisé au niveau des groupes d'étudiants semble avoir répondu aux attentes des apprenants.

Il est également à noter que les deux dispositifs présentés dans cet article n'ont pas été optimaux dès les premières mises en œuvre. E-LearningScape a été déployé à 5 reprises et l'ARE ChemCraft est reconduit chaque année depuis 2014. Le processus est itératif : les premiers résultats permettent d'identifier les améliorations à apporter et permet de faire évoluer les dispositifs pédagogiques en fonction des retours et difficultés rencontrés par les apprenants.

Comme toutes nouvelles initiatives, elles ont demandé un investissement important de la part des enseignants. Dans certains cas, l'aide précieuse des ingénieurs pédagogiques a permis de résoudre les difficultés et d'accompagner les enseignants, par exemple pour l'encadrement des différents groupes de joueurs lors de l'expérimentation d'E-LearningScape. Mais, d'une manière générale, les enseignants s'engagent dans de telles initiatives non pas pour gagner du temps dans leurs enseignements mais parce qu'ils jugent les résultats de l'enseignement plus riches, plus en adéquation avec les attentes des apprenants, et plus précis en termes d'individualisation, de didactique ou de feedback (retour) vers les apprenants. 


\subsection{Retour sur le processus d'innovation pédagogique}

Les enseignants sont les premiers observateurs des difficultés rencontrées par les apprenants et sont les mieux placés pour imaginer des solutions pédagogiques répondant aux besoins des apprenants. Bien que l'enseignement supérieur offre une grande liberté tant sur la forme que sur le fond des enseignements, il n'empêche que les enseignants du supérieur doivent composer avec les contraintes inhérentes aux formations dans lesquelles ils interviennent. En premières années de Licence par exemple, où le nombre d'inscrits peut facilement dépasser le millier d'étudiants, le cadre pédagogique favorise l'uniformisation des enseignements afin que chaque étudiant puisse avoir accès aux mêmes méthodes d'enseignement et d'évaluation. Dans ce contexte, un enseignant volontaire qui souhaiterait proposer à son groupe d'étudiants une approche pédagogique différente ou des modalités d'évaluation différentes créerait de manière incidente une inégalité entre ses étudiants et le reste de la promotion. Le risque dans ce cas serait d'exposer l'équipe pédagogique à des recours juridiques motivés par des différences de traitement non institutionnalisés dans les plaquettes de formation. Pour prévenir ce risque, il est par exemple demandé aux enseignants participant à l'unité d'enseignement d'initiation à la programmation à Sorbonne Université en L1 de suivre le manuel pédagogique édité. Les enseignants peuvent prendre la liberté de traiter tel ou tel exercice mais doivent tous utiliser le même environnement de programmation et dispenser les mêmes évaluations.

Revisiter les approches pédagogiques pour, par exemple, susciter la coopération entre les apprenants et favoriser la motivation, ne relève donc pas uniquement de l'enseignant comme acteur de ses enseignements mais repose surtout sur le cadre qui lui est offert pour innover dans sa pratique. En effet, contrairement aux enseignements en primaire et secondaire régis par des programmes nationaux, le contenu des enseignements dans le supérieur est défini entre pairs et validé par les instances décisionnelles de chaque université. Les équipes pédagogiques peuvent alors se structurer pour définir un cadre propice à la mise en place d'approches pédagogiques différenciées. C'est dans ce cadre qu'ont pu être conçus les ARE proposés à plus de 1500 étudiants chaque année et dont l'ARE ChemCraft n'est qu'un exemple.

Transversalement à l'élaboration de ces parcours de formation définis par les enseignants euxmêmes, les institutions déploient des services pour accompagner les enseignants dans leur démarche de faire évoluer leurs pratiques. Pour le cas de Sorbonne Université, la prise de conscience d'un manque de communication au sujet de l'innovation pédagogique et/ou globalement du « Numérique » a permis de fédérer un certain nombre d'approches isolées. 
Un centre transversal à Sorbonne Université a ainsi été créé. Intitulé CAPSULE (Centre d'Accompagnement à la Pédagogie et Support à l'Expérimentation), il a pour ambition d'accompagner les enseignants à faire évoluer leurs pédagogies grâce à des ressources humaines (ingénieurs pédagogiques, équipe de production vidéo, ingénieurs informatiques) et à leurs compétences. Outre ces moyens humains, le centre est doté également de moyens logistiques : il dispose de locaux d'expérimentation (teaching lab, studio d'enregistrement) et de matériels à disposition des enseignants (table collaborative, casque de réalité virtuelle, écran interactif, boîtiers de vote électronique (clickers)...).

Intermédiaires entre l'université et les enseignants, les UFR (Unité de Formation et de Recherche) peuvent également jouer un rôle important pour inciter les enseignants à dynamiser leurs enseignements. C'est le cas à Sorbonne Université où l'UFR d'Ingénierie organise depuis 2015, les « Midis de la pédagogie » (repris aujourd'hui par CAPSULE). Ces réunions ont pour objectif de permettre à toute personne intéressée par ces questions de réfléchir ensemble sur les pratiques pédagogiques et peuvent susciter/motiver de nouvelles vocations. En effet, si le premier pas est difficile à franchir, coûteux : ces expériences montrent qu'ils ont eu le mérite d'amorcer une démarche qui est reconduite et s'étend chaque année.

CAPSULE permet donc aux enseignants d'échanger sur leurs pratiques, de découvrir et de se former sur ce qui existe au niveau local, national et même international en vue de monter des projets pédagogiques en lien avec leurs problématiques d'enseignement. C'est dans ce cadre que le projet E-LearningScape a été initié.

De l'analyse de ces deux dispositifs pédagogiques (que sont E-LearningScape et l'ARE Chem Craft) et des supports institutionnels en place à Sorbonne Université, nous proposons une illustration du processus d'innovation pédagogique (voir Figure 1). Ainsi, les ressorts permettant aux enseignants de faire évoluer leurs approches pédagogiques s'appuient bien entendu sur les enseignants eux-mêmes qui conçoivent et définissent le cadre des formations mais également sur des impulsions institutionnelles validant les propositions des enseignants et fournissant des services supports aidant les enseignants à préparer, mettre en œuvre et évaluer leurs enseignements à travers l'analyse des retours des apprenants ou l'analyse de leurs traces d'interactions avec des dispositifs numériques (tels que des jeux sérieux par exemple) lors des différentes itérations de mise en œuvre des formations. Le processus d'aide à l'innovation pédagogique à Sorbonne Université repose donc sur deux piliers fondateurs : les enseignants et les services supports fournis par l'institution. 


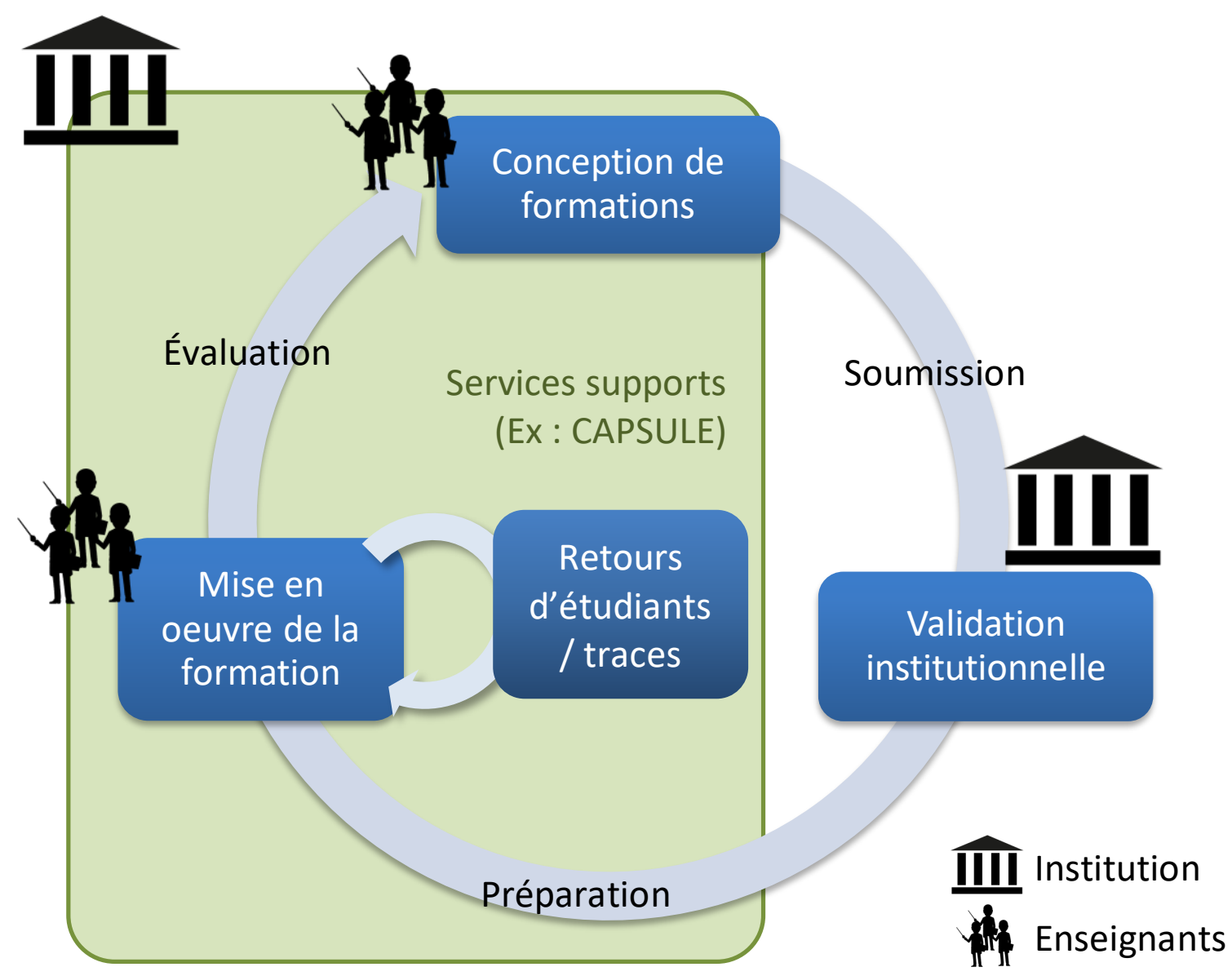

Figure 1 : Processus d'aide à l'innovation pédagogique à Sorbonne Université

\section{Conclusion}

Au travers de deux dispositifs très différents, nous avons vu comment certains enseignants de Sorbonne Université avec l'appui de leur institution repensent leurs enseignements pour proposer des situations pédagogiques stimulantes pour les apprenants. L'analyse des deux dispositifs que sont les ARE (à travers l'exemple de ChemCraft) et la mise en œuvre du jeu ELearningScape nous permet de dégager une illustration du processus d'aide à l'innovation pédagogique.

Les ressorts de motivation mis en lumière sont divers et peuvent même être combinés. Ces deux dispositifs ont en commun leur dimension collaborative voire coopérative autour du concept de jeu sérieux mais exploitent ces composantes différemment. Dans E-LearningScape, les apprenants jouent au jeu et collaborent sur un temps court pour résoudre les énigmes qui leur sont proposées alors que dans ChemCraft, les apprenants coopèrent sur un temps long dans une 
démarche par projet pour concevoir et réaliser leur propre jeu sérieux. Dans E-LearningScape, les apprenants consomment le jeu, alors que dans ChemCraft, ils le produisent.

CAPSULE a investi des moyens financiers dans le développement du jeu E-LearningScape et fournit également des moyens humains (à travers ses ingénieurs pédagogiques) pour seconder les enseignants et aider au déploiement du jeu lors des séances de formations. L’Université, quant à elle, a su offrir aux enseignants un cadre propice à l'innovation pédagogique en L1 au travers des ARE dont ChemCraft n'est qu'un exemple. Ainsi, que ce soit pour E-LearningScape ou ChemCraft, ces initiatives n'auraient pu être rendues opérationnelles sans l'implication fondamentale d'enseignants et le support tout aussi fondamental de leur institution. C'est cette synergie qui a permis de mettre en œuvre les deux projets présentés dans cet article et qui ont pour ambition de proposer des activités adaptées aux attentes actuelles des apprenants.

\section{Références bibliographiques}

Attias-Donfut, C. (1991). Générations et âges de la vie. Paris, Presses Universitaires de France - PUF. 128 p.

Bandura, A. (1986). Social foundations of thought and action: A social cognitive theory. Prentice-Hall.

Cui, Y., Trent, E. S., Sullivan, P. M. et Matiru, G. N. (2003). Cause-related marketing: How generation Y responds. International Journal of Retail \& Distribution Management, 31(6), 310-320. https://doi.org/10.1108/09590550310476012

Elmore, T. (2010). Generation iY: Our last chance to save their future. Poet Gardener Publishing.

Garbarini, D. et Muratet, M. (2020). Accessibilité et jeux vidéo : Quid de l'architecture logicielle Entités-Composants-Systèmes? Handicap 2020, Paris.

Healey, M. et Jenkins, A. (2009). Developing undergraduate research and inquiry. Higher Education Academy.

Marne, B., Huynh-Kim-Bang, B. et Labat, J.-M. (2011). Articuler motivation et apprentissage grâce aux facettes du jeu sérieux. EIAH, 69-80.

McCrindle, M. et Fell, A. (2020). Understanding generation alpha. McCrindle Research.

Miller, F. S. (2012). Transforming learning environments: Strategies to shape the next generation. Emerald.

Mohr, K. A. J. et Mohr, E. S. (2017). Understanding generation Z students to promote a contemporary learning environment. https://doi.org/10.15142/T3M05T

Muratet, M., Arneton, M., Ferrand, M.-H., Geffroy, V., Vanbrugghe, A. et Garbarini, D. (2020). De la réingénierie d'un serious escape game sur la société inclusive aux recherches en informatique. 1024: Bulletin de la Société Informatique de France, 73-81. 
Nacke, L. E., Bateman, C. et Mandryk, R. L. (2011). BrainHex: Preliminary Results from a Neurobiological Gamer Typology Survey. 6972.

Palley, W. (2012). Gen Z: Digital in Their DNA. Wundrman Thompson.

Reilly, P. (2012). Understanding and Teaching Generation Y.

Seemiller, C. et Grace, M. (2016). Generation Z Goes to College. John Wiley \& Sons.

Seemiller, C., et Grace, M. (2017). Generation Z: Educating and engaging the next generation of students. About Campus: Enriching the Student Learning Experience, 22(3), 21-26. https://doi.org/10.1002/abc.21293

Sheahan, P. (2005). Generation Y: Thriving and surviving with Generation Y at work. Hardie Grant Books.

Viau, R. (1997). La motivation en contexte scolaire. De Boeck.Viau, R. (1997). La motivation en contexte scolaire. De Boeck. 